\title{
FORUM
}

\section{Errors of a Simple Doppler Computer due to the Spheroidal Form of the Earth}

\author{
from J. E. D. Williams
}

DOPPLER-operated computers which perform plane D.R. navigation are currently offered to the airlines. A datum track and distance to go are set; from the time of selecting $\mathrm{ON}$, the computer resolves small elements of distance run (input doppler and heading reference) as follows :

Change in across-track dist. = element of dist. run $\times \sin$ (actual tr. - set tr.)

Change in along-track dist. = element of dist. run $\times \cos$ (actual tr. - set tr.) These relations are true for a plane Earth.

The terms across-track distance and along-track distance lack precision on a spheroid: for example the across-track distance could be measured along a geodesic, an orthodrome, or a rhumb line. It seems reasonable to define the terms as follows.

The aircraft's position may be reached by travelling from the starting point along the set rhumb line for the along-track distance and then (turning to the right or left as indicated) travelling along a rhumb-line track at right angles for the across-track distance.

In Fig. I, A and B are positions on a mercator chart before and after flying a small element of distance $\Delta$ salong a track $\theta$. $\mathrm{AC}$ is the distance run parallel to the datum track $\theta_{0} . P$ and $Q$ are, on the chart, at the feet of perpendiculars from $A$ and $C$ to the datum track $\theta_{0} . a, b, c$, $p, q$, are the points on the terrestrial spheroid corresponding to $\mathrm{A}, \mathrm{B}, \mathrm{C}, \mathrm{P}$, Q.

Clearly,

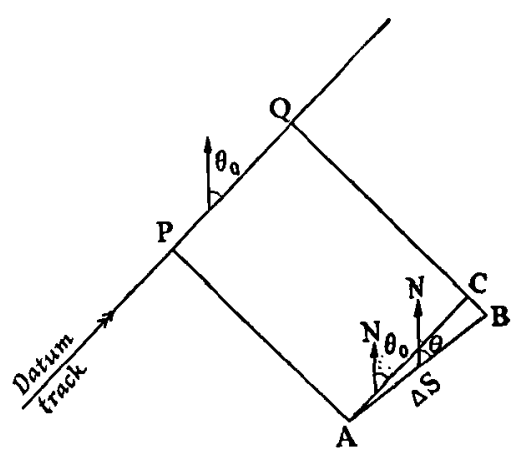

Fig. 1

Computer change of across-track distance

$$
=\mathrm{cb}=\Delta s \sin \left(\theta-\theta_{0}\right) \text {. }
$$

Actual change of across-track distance

Error in change of across-track distance

$$
=\mathrm{qb}-\mathrm{pa}=\Delta s \sin \left(\theta-\theta_{0}\right)+\mathrm{qc}-\mathrm{pa} .
$$

$$
=\mathrm{qc}-\mathrm{pa}
$$

Computer change of along-track distance $=\mathrm{ac}=\Delta s \cos \left(\theta-\theta_{0}\right)$ where

Actual change of along-track distance $=\mathrm{pq}=\mathrm{ac} r_{\mathrm{a}} / \mathrm{r}_{\mathrm{p}}$

$$
\begin{aligned}
& r_{\mathrm{a}}=\text { chart scale at a } \\
& r_{\mathrm{p}}=\text { chart scale at } \mathrm{p} \text { (since } \mathrm{PQ}=\mathrm{AC} \text { ). }
\end{aligned}
$$

Let $m$ be the distance of a point, measured along its meridian, from the equator. It may be shown that the rhumb-line distance between two points of 
meridional distance $m_{1}, m_{2}$ is $\left(m_{1}-m_{2}\right)$ sec $\alpha$ on a spheroid, $\alpha$ being the track. Hence, if the meridional distances of a, c, p, q are respectively $m_{\mathrm{a}}, m_{\mathrm{c}}, m_{\mathrm{p}}, m_{\mathrm{q}}$, Error in across-track distance $=q c-p a$

in along-track distance $=p q-a c$

$$
=\left[\left(m_{\mathrm{q}}-m_{\mathrm{c}}\right)-\left(m_{\mathrm{p}}-m_{\mathrm{a}}\right)\right] \operatorname{cosec} \theta_{0}
$$

$$
=\left[\left(m_{\mathrm{q}}-m_{\mathrm{p}}\right)-\left(m_{\mathrm{c}}-m_{\mathrm{a}}\right)\right] \sec \theta_{0}
$$

Hence the total change in error is in an east/west direction and is

$$
\left[\left|m_{\mathrm{q}}-m_{\mathrm{c}}\right| \sim\left|m_{\mathrm{p}}-m_{\mathrm{a}}\right|\right] \sec \theta_{0} \operatorname{cosec} \theta_{0}
$$

The scale factor, $r$ in (2) may be written as $f(m)$, a function of meridional distance.

Then to the first order of accuracy

$$
\begin{aligned}
\mathrm{I}-\frac{r_{\mathrm{a}}}{r_{\mathrm{p}}} & =\frac{d \mathrm{r}}{d m} \frac{m_{\mathrm{p}}-m_{\mathrm{a}}}{r_{\mathrm{p}}}=\frac{f^{\prime}(m)}{f(m)}\left(m_{\mathrm{p}}-m_{\mathrm{a}}\right) \\
& =\frac{f^{\prime}(m)}{f(m)} \times \text { across-track distance } \times \sin \theta_{0}
\end{aligned}
$$

Hence from (2), (3) and (4), increment in total error, in a west/east direction, $=\Delta s \cos \left(\theta-\theta_{0}\right) \frac{f^{\prime}(m)}{f(m)} \times$ across-track distance

The quantity $100 f^{\prime}(m) / f(m) \times$ across-track distance is shown graphically in Fig. 2

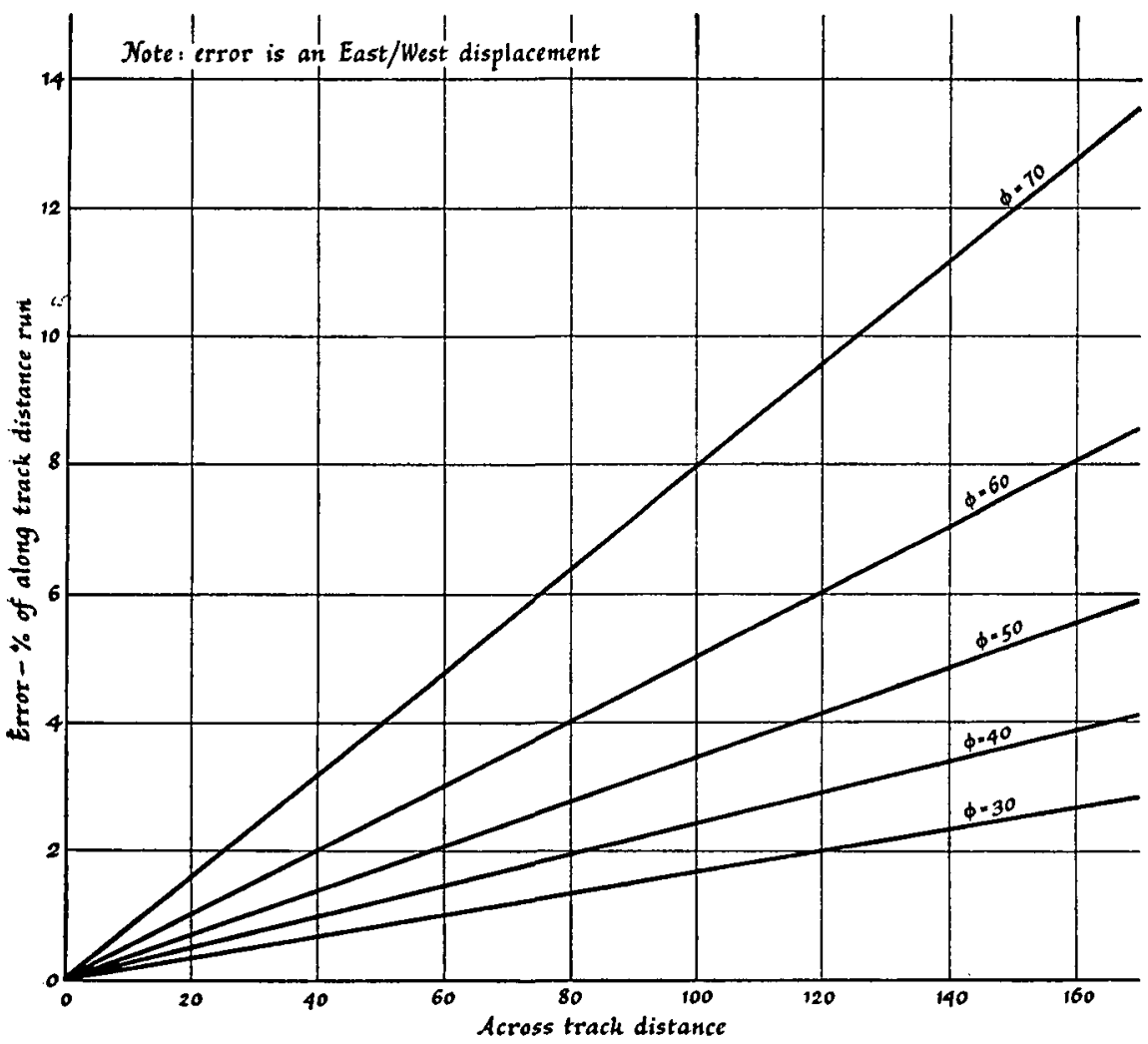

Fig. 2 
against latitude and across-track distance. It represents the increase in total error as a percentage of distance run when the across-track distance is not changing rapidly.

If $X$ is along-track distance and $Y$ is across-track distance, total error in an east/west direction

$$
=\int_{0}^{X} Y \frac{f^{\prime}(m)}{f(m)} d X
$$

For a sphere,

$$
\frac{f^{\prime}(m)}{f(m)}=\frac{\tan \varphi}{a}
$$

where $\varphi=$ latitude and $a=$ radius of the Earth.

Therefore the approximate total error in computer position on a sphere is

$$
\frac{1}{a} \int_{0}^{X} Y \tan \phi d X
$$

This correction could be incorporated in the computer if heading references justified the requirement for accuracy.

\title{
Calculation of Charts on an Oblique Gnomonic Projection by Electronic Computer
}

\author{
from P. B. Sarson
}

(Meteorological Office)

IN the Meteorological Office, great accuracy is not usually attainable in determining or forecasting the position of significant weather features. Special projections of charts are therefore not often required; the normal conic projection with two standard parallels or (near the equator) the mercator projection is quite adequate. However, in the radiolocation of thunderstorms a chart drawn on a gnomonic projection is required. The bearings of each lightning flash within one or two thousand miles are recorded from a small number of special stations (sFerics stations). When these bearings are plotted on a gnomonic chart by straight lines drawn from the appropriate observing station the coordinates of the source of the lightning can be quickly determined and reported through normal meteorological channels. Speed is essential and therefore the charts on which the bearings are drawn are specially designed with the SFERICS stations grouped more or less evenly about the tangential point of the projection plane of the gnomonic chart.

Direction roses are marked in special borders (one for each station) round the edges of the chart so that the bearings can be plotted instantly using a length of cord drawn through a small hole in the chart, at the position of the station, to the appropriate bearing given in the direction-rose border.

Several of these charts have been calculated by pencil-and-paper methods as the demand for a chart for any particular area arose, although none covering so 